\title{
Sensitivity analysis of heat transfers in an asymmetrically heated turbulent channel flow
}

\author{
Martin David ${ }^{* a}$, Adrien Toutant ${ }^{\mathrm{a}}$, and Françoise Bataille ${ }^{\mathrm{a}}$ \\ ${ }^{a}$ Laboratoire PROMES-CNRS (UPR 8521), Université de Perpignan via Domitia, Technosud-Rambla de la thermodynamique, 66100 \\ Perpignan - France
}

\begin{abstract}
A sensitivity analysis of heat transfers in an asymmetrically heated turbulent channel flow is performed using a dedicated heat transfer correlation. The investigated correlation is developed to study the heat transfers between the fluid and the wall in gas-pressurized solar receivers of concentrated solar power tower. The working conditions correspond to high-temperature levels and high heat fluxes. The correlation of the Nusselt number depends on five parameters: the Reynolds number, the Prandtl number, the fluid temperature, the hot and cold wall temperatures. We investigate the sensitivity of the heat flux to the wall and fluid temperatures. The results obtained with the global uncertainty management are compared to direct computations of the errors of measurement. In the global uncertainty management, the heat flux sensitivity is studied with the Taylor expansion of the function. This method assumes the quasilinearity and the quasi-normality of the function; therefore, only small variations of parameters are computed. The study points out the importance of the temperature measurement accuracy for the heat flux evaluation in asymmetrically heated turbulent channel flow. In particular, the results show that the cold wall heat flux is very sensitive to the variations of the cold wall temperature and the bulk temperature of the fluid. The hot wall is less influenced by the temperature variations than the cold wall. The global uncertainty management produces satisfying results on the prediction of the error linked to the uncertainties on bulk temperature. Nevertheless, the hot and cold wall temperature uncertainty propagation are poorly estimated by the method.
\end{abstract}

\section{Nomenclature}

\begin{tabular}{|l|r|}
\hline $\mathrm{T}_{\mathrm{m}}$ & wall temperature average $[\mathrm{K}]$ \\
\hline Greek symbols \\
\hline$\lambda$ & thermal conductivity $[\mathrm{W} / \mathrm{m} / \mathrm{K}]$ \\
\hline$\phi$ & heat flux $\left[\mathrm{W} / \mathrm{m}^{2}\right]$ \\
\hline Subscripts & cold \\
\hline c & hot \\
\hline h &
\end{tabular}

\section{Introduction}

In applied engineering systems, there are many possible sources of uncertainty: measurement such as finite instrument resolution, inexact values of measurement standards, approximations and assumptions incorporated in the measurement method and procedure [1]. The uncertainties may become very problematic when moving from principles to a functioning device. They should be put into perspective with a sensitivity analysis. Indeed, while some systems are poorly affected by parameter variations, some are very sensitive to specific parameters. For instance, in gas turbines, it is generally accepted that a variation of $20 \mathrm{~K}$ in the metal reduces the life of the components by $50 \%$ [2].

Uncertainties can also make difficult the understanding of the results of experiments or numerical simulations. Several authors propose methods to bring uncertainties out. Some works dealing with uncertainties in the fields of fluid mechanics and heat transfers are addressed below. Oliver et al. [3] estimate uncertainties in statistics computed from Direct Numerical Simulation (DNS). They provide a systematic and unified approach for estimating the uncertainties due to finite statistical sampling and the discretization of the Navier-Stokes equations. Carneval et al. [4] propose a stochastic method for heat transfer prediction using Large Eddy Simulation (LES). It consists in coupling a classical uncertainty quantification to LES in a duct with pin fins. The authors prove that the uncertainties related to the unknown conditions, named aleatoric uncertainties, and those related to the physical model, named epistemic uncertainties, are strongly interconnected. Menberg et al. [5] scrutinize three

\footnotetext{
* Corresponding author: martin.david@promes.cnrs.fr
} 
methods for sensitivity analysis in relation to dynamic, high-order, non-linear behaviour and the level of uncertainty in building energy models.

Estimation of uncertainties in heat transfer coefficients determination is a commonly studied topic. For instance, a large number of works tackles the uncertainty propagation when estimating the convection coefficients in a wide spectrum of convective heat transfer processes with the Wilson plot method. Uhía et al. [6] detail the application of the Global Uncertainty Management (GUM) [1] for calculating the uncertainty associated with experimental heat transfer data obtained thanks to the Wilson plot method. They use the specific process of condensation of R-134a on a horizontal smooth tube as an illustration. The influence of the uncertainty of measurements is discussed on the basis of two normalized coefficients proposed by Coleman and Steele [7]: the uncertainty magnification factor (UMF) and the uncertainty percentage contribution (UPC). Wójs and Tietze [8] also study the effects of the temperature interference on the results obtained using the Wilson plot technique. The authors highlight the importance of using adequate experimental data to obtain reliable results.

The Global Uncertainty Management (GUM) establishes general rules for evaluating and expressing uncertainty in measurement that are intended to be applicable to a broad spectrum of measurements. It is considered as a general cross-disciplinary standard, applicable to all fields. The method is detailed in [1]. Studying convective heat transfer, Håkansson [9] notices some incoherence in the literature when regarding heat transfer coefficient values. Using the GUM, he points out that some methods used to determine the heat transfer coefficient are very dependent of the accuracy of measurements. This could be responsible for the various results obtained in the literature.

Heat transfer correlation are widely used in engineering field to estimate wall heat flux of complex devices such as heat exchangers [10]-[12], automobile radiators [13], and solar receivers of concentrated solar power tower [14]. Correlations permit to estimate the heat transfer within, generally, $10 \%$ to $20 \%$ of error [14]-[20]. This accuracy is usually acceptable for predimensioning. However, it is necessary to consider the uncertainty of measurements and their propagation to provide an error range associated with the heat flux prediction. Correlation may also be useful to quantify the heat transfer sensitivity to parameters. For instance, Driscoll and Landrum [21] study uncertainty on heat transfer correlations for fuel in copper tubing. They quantify the influence of uncertainties on the engine design. Scariot et al. [22] analyze the influence of the uncertainty of measurements on the fluid temperature and enthalpy in $\mathrm{CO} 2$ tube flow using heat transfer correlations.

In this paper, a correlation developed for asymmetrically heated turbulent channel flow is used to quantify the uncertainty propagation. The studied correlation is presented in the first part. Then, the sensitivity of the correlation to flow parameters is analyzed in the working conditions of gas-pressurized solar receivers of concentrated solar power tower in the second part. The uncertainty propagation estimated by the GUM is assessed.

\section{Correlation for heat transfer in asymmetrically heated channel flow}

\subsection{Description of the correlation}

The correlation proposed by David et al. [14] aims to estimate the heat transfer in the working conditions of gas-pressurized solar receivers. The correlation has been established thanks to 70 Large Eddy Simulations (LES), leading to a consequent and reliable sample of data. In those LES, the Navier-Stokes equations are solved under the low Mach number approximation in a bi-periodic channel flow. The coupling between velocity and temperature is considered. The gravity force is not taken into account since its impact is negligible in the working conditions. Indeed, the Richardson number is about 10-5. The small turbulent scales are not solved in LES. Their effects on large turbulent structures are considered thanks to the Anisotropic-Minimum-Dissipation (AMD) model. This model has showed good results in similar conditions in [23]-[25]. The proposed correlation is given by equation 1:

$$
\begin{aligned}
& N u_{w}=0.024 \operatorname{Re}_{b}^{0.8} \operatorname{Pr}_{b}^{0.4}\left(\frac{T_{w}}{T_{b}}\right)^{-0.9} \\
& \left(\frac{T_{w}}{T_{w}-T_{b}}\right)^{1.4\left(1-\frac{T_{w}}{T_{m}}\right) \frac{T_{b}}{T_{w}}}
\end{aligned}
$$

Where $T_{w}$ is the wall temperature, $T_{b}$ is the bulk temperature, and $T_{m}$ is the averaged wall temperature. A term accounting for asymmetric heating conditions is added in the equation to reproduce the heat transfers at hot and cold walls. Notice that this term involves 
variables in the exponent. The singularity of the Nusselt number, encountered when the bulk temperature tends towards the cold wall temperature and explained in [26], is reproduced thanks to the difference of temperature at the denominator of the asymmetric group. This term, accounting for asymmetric condition, is equal to 1 in case of symmetric heating conditions.

\subsection{Applicability domain of the correlation}

The proposed correlation is investigated in the commonly encountered working conditions of heat exchangers and solar receivers. See the applicable domain of the correlation in terms of Reynolds number and temperature ranges in Table 1. The wall temperatures range from $293 \mathrm{~K}$ to $1300 \mathrm{~K}$. The fluid temperature varies from $342 \mathrm{~K}$ to $1237 \mathrm{~K}$, Prandtl number is between 0.76 and 3.18 , and heat fluxes are between $4 \mathrm{~kW} / \mathrm{m} 2$ and $578 \mathrm{~kW} / \mathrm{m} 2$.

Table 1. Applicable domain of the correlation

\begin{tabular}{cc}
\hline Symmetric heating & Asymmetric heating \\
\hline $12000<\mathrm{Re}_{\mathrm{b}}<177000$ & $10600<\mathrm{Re}_{\mathrm{b}}<145000$ \\
$0.47<\mathrm{T}_{\mathrm{b}} / \mathrm{T}_{\mathrm{w}}<0.99$ & $1.1<\mathrm{T}_{\mathrm{h}} / \mathrm{T}_{\mathrm{c}}<2.0$ \\
& $0.63<\mathrm{T}_{\mathrm{b}} / \mathrm{T}_{\mathrm{c}}<0.95$ \\
& $0.44<\mathrm{T}_{\mathrm{b}} / \mathrm{T}_{\mathrm{h}}<0.85$ \\
\hline
\end{tabular}

\subsection{Validation of the correlation}

All the Nusselt numbers estimated with the used correlation have a relative error inferior to $9.1 \%$ and more than three-quarters of them are in the error bound $5 \%$. The determination coefficient is 0.993 . The mean error is $0.29 \%$ and standard deviation is $4.1 \%$. In

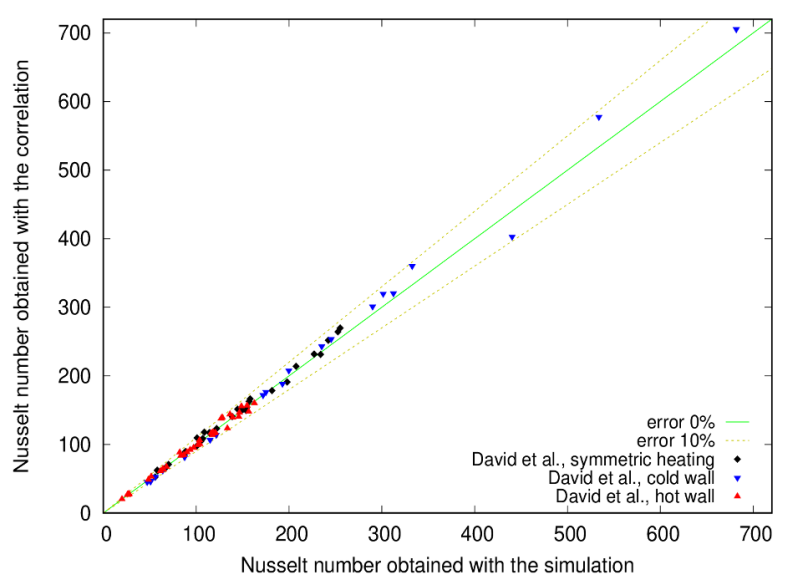

Fig.1. Nusselt numbers obtained with the correlation depending on the Nusselt numbers obtained by the simulations.

Figure 1, The Nusselt numbers obtained with the correlation proposed by David et al. [14] are plotted as function of the Nusselt numbers obtained with the numerical simulations. Black dots stand for the Nusselt numbers obtained under symmetric heating conditions. Red dots, respectively blue dots, account for the Nusselt numbers obtained at the hot wall, respectively cold wall, under asymmetric heating conditions. The Nusselt number range is between 20 and 680. The results show that all the points are between the line representing $10 \%$ of error.

\section{Sensitivity of the correlation}

An way interesting to highlight the influence of each parameter on the wall heat flux is to perform a sensitivity study using the correlation. It gives the measurement accuracy necessary to provide reliable estimation of the wall heat fluxes. In its applicable domain, the sensitivity of the correlation reflects the physic sensitivity. A high temperature sensitivity of the correlation means that the physical wall heat flux is, also, greatly impacted by this parameter. In this study, the sensitivity of the correlation is analyzed with two methods. The computation of the propagation of uncertainties using the GUM method is compared to the results obtained by the direct calculation of heat fluxes with various temperature values.

\subsection{Working conditions}

The sensitivity of the wall heat fluxes are studied in the typical working conditions of gas-pressurized solar receivers. These conditions are summarized in Table 2.

Table 2. Studied conditions for the sensitivity study

\begin{tabular}{cccc}
\hline $\mathrm{Re}_{\mathrm{b}}$ & $\mathrm{Pr}_{\mathrm{b}}$ & $\mathrm{T}_{\mathrm{h}}[\mathrm{K}]$ & $\mathrm{T}_{\mathrm{c}}[\mathrm{K}]$ \\
\hline 60000 & 0.87 & 1300 & 900 \\
\hline
\end{tabular}

The wall heat fluxes are computed using the Nusselt numbers obtained with the correlation thanks to equation 2 :

$$
\phi_{w}=\frac{\lambda_{w}\left(T_{w}-T_{b}\right) N u_{w}}{L}
$$

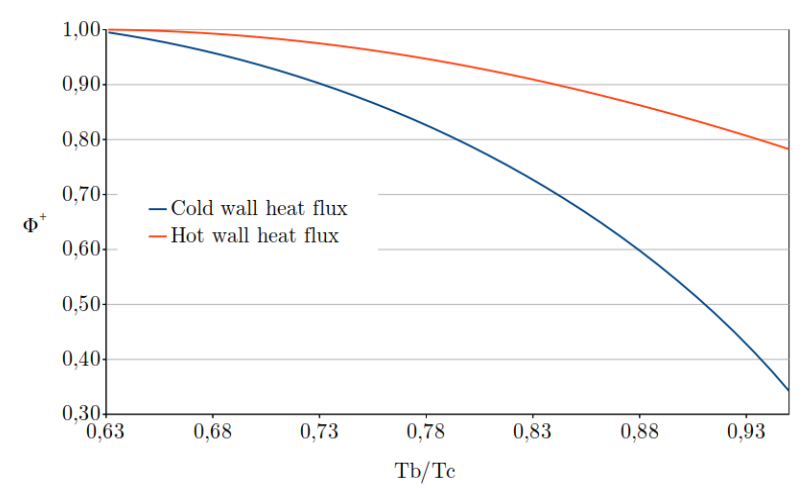

Fig 2: Wall heat transfer as a function of the bulk-to-wall temperature ratio. 
In the studied conditions and considering the minimum ratio between bulk temperature and cold wall temperature, the heat transfer at the cold wall is 170 $\mathrm{kW} / \mathrm{m} 2$ and the transfer at the hot wall is $265 \mathrm{~kW} / \mathrm{m} 2$. The wall heat flux is normalized by the biggest wall heat transfer obtained, i.e. for a bulk-to-cold wall temperature ratio of 0.63 . The normalized heat fluxes are decreasing as the bulk temperature is getting closer to the cold wall temperature. As expected, the cold wall heat flux is more sensitive to the evolution of the bulk temperature than the hot wall heat flux.

\subsection{Global Uncertainty Management}

The GUM provides an analytic expression of the heat flux uncertainties as a function of the flow parameters and the uncertainty of measurements. The uncertainty propagation on the wall heat fluxes are obtained by applying the GUM to equation 2 .

$$
\frac{\Delta \phi}{\phi}=\sqrt{A^{2}+B^{2}+C^{2}+D^{2}+E^{2}}
$$

With

$$
\begin{aligned}
& A=\Delta R_{b}\left(\frac{0.8}{R e_{b}}\right) \\
& B=\Delta \operatorname{Pr}_{b}\left(\frac{0.4}{P r_{b}}\right) \\
& C=\Delta T_{b}\left(\frac{0.9}{T_{b}}+\frac{1.4}{T_{w}} \alpha(\gamma+\log (\beta))-\frac{1}{T_{w}-T_{b}}\right) \\
& D=\Delta T_{w}\left(\frac{0.9}{T_{w}}\right)+\Delta T_{w}\left(1.4 \frac{\alpha T_{b}}{T_{w}}\left(\gamma+\log (\beta)\left(1+\frac{1}{\delta}+\frac{\alpha}{\delta}\right)\right)\right. \\
& -\Delta T_{w}\left(\frac{1}{T_{w}-T_{b}}\right) \\
& E=\Delta T_{\bar{w}}\left(2.8 \frac{T_{b}}{\left(T_{w}+T_{\bar{w}}\right)^{2}} \log (\beta)\right)
\end{aligned}
$$

Where

$$
\begin{aligned}
& \alpha=\frac{T_{\bar{w}}-T_{w}}{T_{w}+T_{\bar{w}}} ; \beta=\frac{T_{w}}{T_{w}-T_{b}} ; \gamma=\frac{T_{b}}{T_{w}-T_{b}} ; \\
& \delta=\frac{T_{\bar{w}}-T_{w}}{T_{w}}
\end{aligned}
$$

Here, the symbol designs the opposite wall. For instance, is the uncertainty measurement on the bulk temperature. The uncertainty associated with the thermal conductivity has almost no influence on the results. It is then, neglected to simplify the equation. In the analysis, the uncertainties on the input variables are assumed to be uncorrelated. In the following, we consider uncertainty of measurements on one parameter at a time and we only study the uncertainties relative to temperature.

\subsection{Influence of the bulk-to-wall temperature ratio}

The uncertainties on the wall heat fluxes are not only dependent on the uncertainty of measurements: the working flow conditions significantly affect the propagation of uncertainties. In the following, the results of the GUM analysis and the direct calculation of the heat flux are plotted as functions of the ratio of bulk and cold wall temperatures in applicable domain of the correlation. In the next, this ratio is called bulk-to-wall temperature ratio for practical reasons. Five uncertainty of measurements are studied: $2 \%, 4 \%, 6 \%, 8 \%$, and $10 \%$.

\subsubsection{Influence of the bulk-to-wall temperature ratio on the propagation of wall temperature uncertainties}
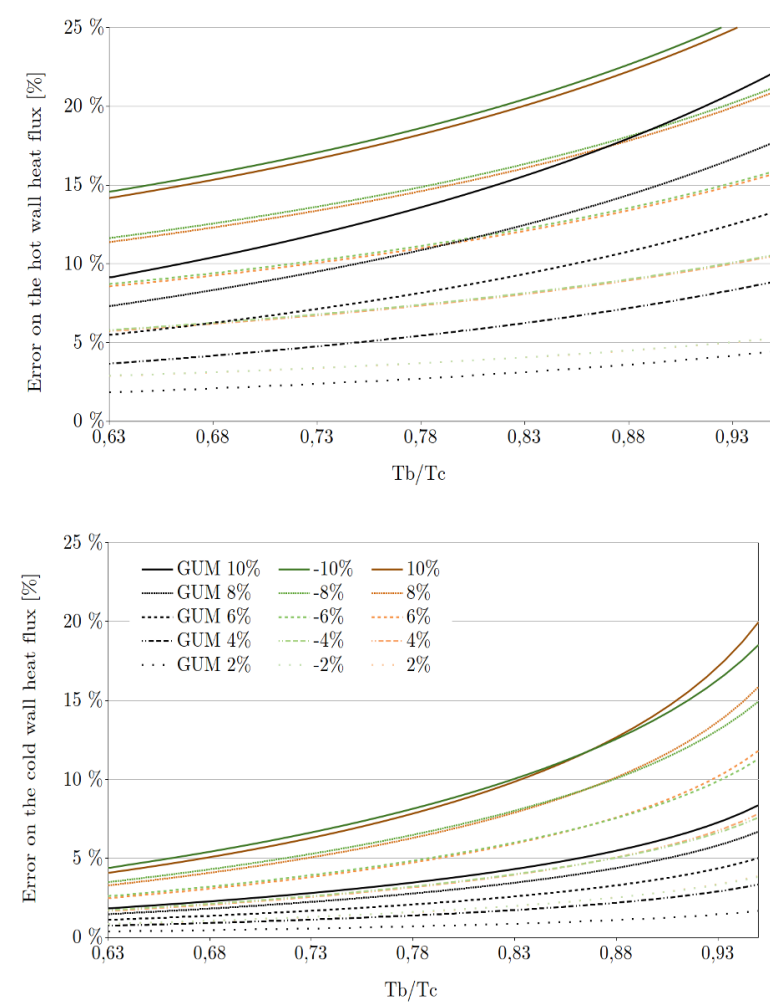

Fig 3. Influence of the uncertainty of measurements on the hot wall temperature as a function of the bulk-to-wall temperature ratio. The top graph, respectively bottom graph, displays the errors committed on the hot wall heat flux, respectively cold wall heat flux.

The effect of the hot wall temperature uncertainty of measurements on the wall heat fluxes is observed in Figure 3. The results show that the error on the wall heat fluxes is increasing with the bulk-to-wall 
temperature ratio. However, the associated heat fluxes are lower, as seen on figure 2. For the same temperature ratio, improving the measurement accuracy permits to shift the strong increase of the uncertainties to bigger bulk-to-wall temperature ratio. The uncertainty propagation is quasi-normal since the overestimation and the underestimation of the hot wall temperature lead to similar errors on the heat flux. The results show that the cold side is more impacted by the uncertainty of measurements than the hot side. The

error profiles provided by the GUM are comparable to the ones obtained with the direct calculation. However, it underestimates the error committed on both walls. The GUM is more accurate when the uncertainty measurement is low. Indeed, as the studied function are not linear, the error committed by the GUM increases with the uncertainty value. For instance, at a bulk-towall temperature ratio of 0.83 , a measurement of the
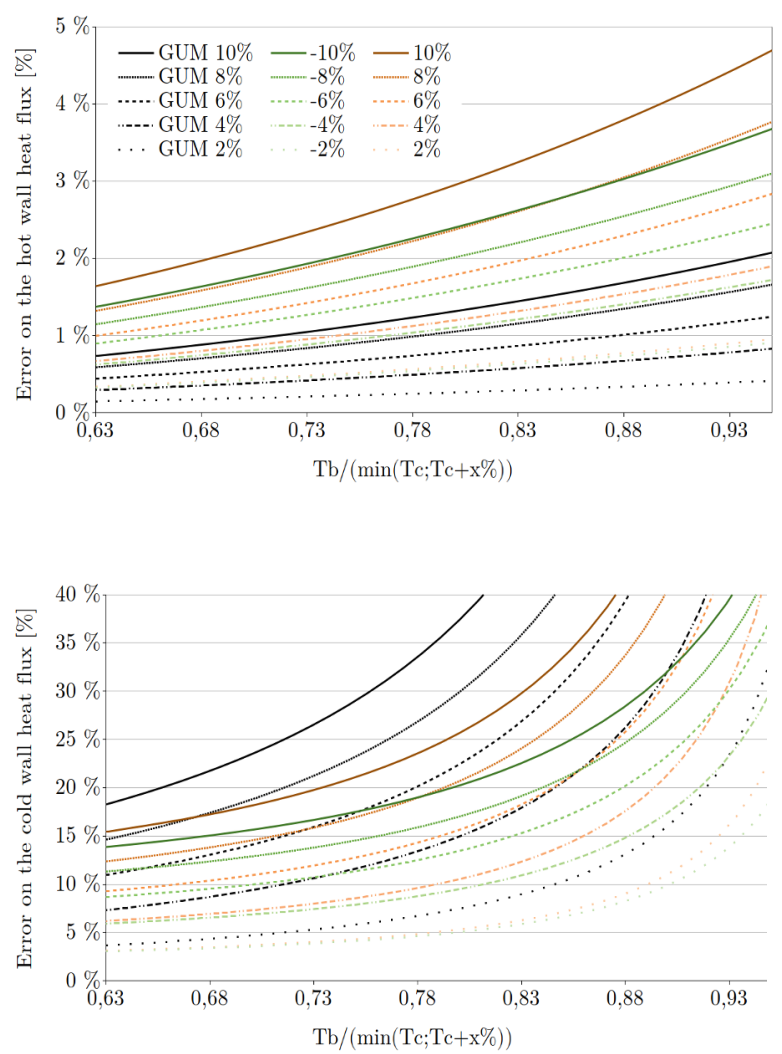

Fig 4. Influence of the uncertainty of measurements on the cold wall temperature as a function of the bulk-to-wall temperature ratio. The top graph, respectively bottom graph, displays the errors committed on the hot wall heat flux, respectively cold wall heat flux.

hot wall temperature with $10 \%$ of error leads to an error of $20 \%$ while the GUM predicts an error of $15 \%$. A measurement of the hot wall temperature with $2 \%$ of error leads to an error of $4 \%$ while the GUM predicts an error of $3 \%$. The influence of the cold wall uncertainty measurement on the wall heat fluxes is plotted on figure 4 . The errors are plotted as functions
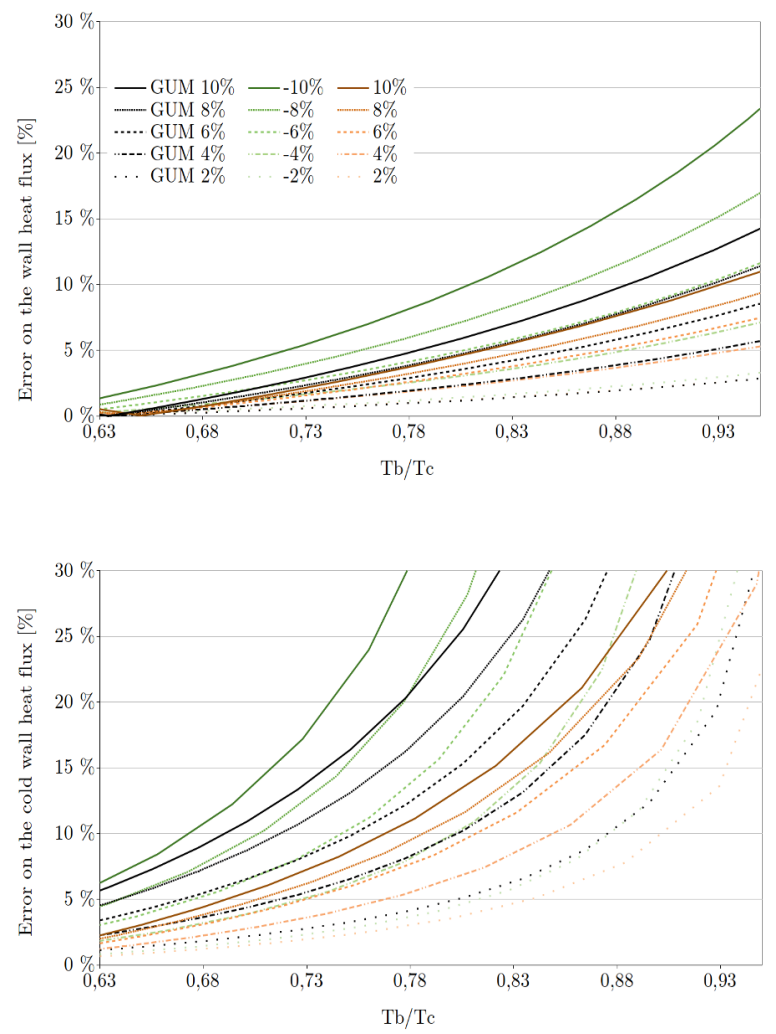

Fig 5. Influence of the uncertainty of measurements on the bulk temperature as a function of the bulk-to-wall temperature ratio. The top graph, respectively bottom graph, displays the errors committed on the hot wall heat flux, respectively cold wall heat flux.

of the ratio between the bulk temperature and the minimum value of the cold wall in order to respect the applicable domain of the correlation. Indeed, in the case of an underestimation of the cold wall, the underestimated temperature is used to compute the ratio. In the case of an overestimation of the cold wall temperature, the reference cold wall temperature is used since it is limiting the validity domain of the correlation. As observed on figure 3, the error on the heat transfers increases with the bulk-to-wall temperature ratio. For the same uncertainty measurement on the cold wall temperature, the error on the hot wall heat flux is significantly lower than the error on the cold wall heat flux. The error increases quasi-linearly at the hot wall whereas it is soaring at the cold wall. The error on the heat transfer is clearly not symmetric on both sides. For instance, at the cold wall, for a temperature ratio of 0.8 and a uncertainty measurement of $10 \%$, the results show an error of $26 \%$ in the case of an overestimation and an error of $20 \%$ in the case of an underestimation. The GUM underestimates the error at the hot wall and overestimates it at the cold wall. As observed on figure 3 , the lowest the uncertainty measurement is, the most accurate the GUM is. 


\subsubsection{Influence of the bulk-to-wall temperature ratio on the propagation of bulk temperature uncertainties}

The influence of the uncertainty of bulk temperature measurement on the wall heat fluxes is observed on figure 5. Once again, the same behavior as seen on figure 3 and 4 are highlighted: the hot wall heat flux is less impacted by the uncertainties on the bulk temperature than the cold wall heat flux. At the hot wall, the error on the heat transfer increases almost linearly whereas at the cold side the error soars as the bulk-to-wall temperature ratio get closer to 0.95 . Accurate measurements permit to keep reasonable error on the hot wall heat flux even for high values of the temperature ratio. At the cold side, even a $2 \%$ uncertainty on the bulk temperature causes an important error on the cold wall heat flux. The GUM produces a quite satisfying estimation knowing that it assumes a normal distribution of the error. Indeed, the error predicted with the GUM is between the error obtained for an underestimation and the error obtained for an overestimation.

\section{Conclusions}

In this paper, the correlation proposed by David et al. for asymmetrically heated turbulent channel flow has been presented. Then, a sensitivity study of the correlation to flow parameters has been carried out in the working conditions of gas-pressurized solar receivers of concentrated solar power tower. The entire range of the applicable domain in terms of bulk-to-wall temperature ratio has been investigated.

The results show that the closer the cold wall temperature and the bulk temperature are, the bigger the uncertainty propagation is. At the same time, the wall heat flux decreases which reduce the importance of the committed error. The cold wall heat flux is more sensitive to the wall and bulk temperature variations than the hot wall heat flux. Overestimation and underestimation of the hot wall temperature produces similar error profiles indicating a normal distribution of the error. Uncertainties on the cold wall and on the bulk temperatures induce asymmetric errors. The GUM provides an analytic expression of the uncertainties associated to the wall heat transfers. Since it assumes a linear distribution of the error, the GUM provides more accurate results for small uncertainties. This method produces satisfying results on the prediction of the error linked to the uncertainties on bulk temperature. However, it underestimates the error committed by uncertainties on the hot wall temperature as well as the error committed on the hot wall heat flux when the uncertainties are associated with the cold wall temperature.

\section{Acknowledgments}

The authors gratefully acknowledge the CEA for the development of the TRUST platform. This work was granted access to the HPC resources of CINES under the allocation 2019-A0062A05099 and 2020A0082A05099 made by GENCI. The authors also acknowledge the Occitania region for their funding of the thesis grant.

\section{References}

1. JCGM, "Evaluation of measurement data - Guide to the expression of uncertainty in measurement," 2008. https://www.bipm.org/en/publications/guides/gum.html (accessed Jan. 08, 2021).

2. J. Han, S. Dutta, and S. V. Ekkad, Gas Turbine Heat Transfer and Cooling Technology. CRC Press - Taylor \& Francis Group, 2012.

3. T. A. Oliver, N. Malaya, R. Ulerich, and R. D. Moser, "Estimating uncertainties in statistics computed from direct numerical simulation," Phys. Fluids, vol. 26, no. 3, p. 035101, Mar. 2014

4. M. Carnevale, F. Montomoli, A. D'Ammaro, S. Salvadori, and F. Martelli, "Uncertainty Quantification: A Stochastic Method for Heat Transfer Prediction Using LES,” J. Turbomach., vol. 135, Jun. 2013.

5. K. Menberg, Y. Heo, and R. Choudhary, "Sensitivity analysis methods for building energy models: Comparing computational costs and extractable information," Energy Build., vol. 133, pp. 433-445, Dec. 2016.

6. F. Uhía, A. Campo, and J. Fernández-Seara, "Uncertainty analysis for experimental heat transfer data obtained by the Wilson plot method: Application to condensation on horizontal plain tubes," Therm. Sci., vol. 17, Jan. 2013.

7. G. N. Coleman, J. Kim, and R. D. Moser, "A numerical study of turbulent supersonic isothermalwall channel flow," J. Fluid Mech., vol. 305, pp. 159183, Dec. 1995.

8. K. Wójs and T. Tietze, "Effects of the temperature interference on the results obtained using the Wilson plot technique," Heat Mass Transf., vol. 33, no. 3, pp. 241-245, Dec. 1997.

9. A. Håkansson, "An investigation of uncertainties in determining convective heat transfer during immersion frying using the general uncertainty management framework," J. Food Eng., vol. 263, pp. 424-436, Dec. 2019. 
10. S. G. Penoncello, Thermal Energy Systems: Design and Analysis. CRC Press - Taylor \& Francis Group, 2015.

11. D. Taler, "Mathematical modeling and control of plate fin and tube heat exchangers," Energy Convers. Manag., vol. 96, pp. 452-462, May 2015.

12. M. Trojan and D. Taler, "Thermal simulation of superheaters taking into account the processes occurring on the side of the steam and flue gas," Fuel, vol. 150, pp. 75-87, Jun. 2015.

13. F. W. Dittus and L. M. K. Boelter, "Heat transfer in automobile radiators of the tubular type," Int. Commun. Heat Mass Transf., vol. 12, no. 1, pp. 3-22, Jan. 1985.

14. M. David, A. Toutant, and F. Bataille, "Numerical development of heat transfer correlation in asymmetrically heated turbulent channel flow," Int. J. Heat Mass Transf., vol. 164, p. 120599, Jan. 2021.

15. E. N. Sieder and G. E. Tate, "Heat Transfer and Pressure Drop of Liquids in Tubes," Ind. Eng. Chem., vol. 28, no. 12, pp. 1429-1435, Dec. 1936.

16. A. P. Colburn, "A method of correlating forced convection heat-transfer data and a comparison with fluid friction,” Int. J. Heat Mass Transf., vol. 7, no. 12, pp. 1359-1384, Dec. 1964.

17. E. Battista and H. C. Perkins, "Turbulent heat and momentum transfer in a square duct with moderate property variations," Int. J. Heat Mass Transf., vol. 13, no. 6, pp. 1063-1065, Jun. 1970.

18. J. Ma, Longjian. Li, Y. Huang, and X. Liu, "Experimental studies on single-phase flow and heat transfer in a narrow rectangular channel," Nucl. Eng. Des., vol. 241, no. 8, pp. 2865-2873, Aug. 2011.

19. V. Gnielinski, "On heat transfer in tubes," Int. J. Heat Mass Transf., vol. 63, pp. 134-140, Aug. 2013.

20. D. Taler, "Simple power-type heat transfer correlations for turbulent pipe flow in tubes," J. Therm. Sci., vol. 26, no. 4, pp. 339-348, Aug. 2017.

21. E. Driscoll and D. Landrum, "Uncertainty Analysis on Heat Transfer Correlations for RP-1 Fuel in Copper Tubing," NTRS - NASA Technical Reports Server, 2004.

22. V. K. Scariot, G. M. Hobold, and A. K. da Silva, "On the sensitivity to convective heat transfer correlation uncertainties in supercritical fluids," Appl. Therm. Eng., vol. 145, pp. 123-132, Dec. 2018.
23. D. Dupuy, A. Toutant, and F. Bataille, "A posteriori tests of subgrid-scale models in an isothermal turbulent channel flow," Phys. Fluids, vol. 31, no. 4, p. 045105, Apr. 2019.

24. D. Dupuy, A. Toutant, and F. Bataille, "A posteriori tests of subgrid-scale models in strongly anisothermal turbulent flows," Phys. Fluids, vol. 31, no. 6, p. 065113, Jun. 2019.

25. D. Dupuy, A. Toutant, and F. Bataille, "A priori tests of subgrid-scale models in an anisothermal turbulent channel flow at low mach number," Int. J. Therm. Sci., vol. 145, p. 105999, Nov. 2019.

26. D. A. Nield, "Forced convection in a parallel plate channel with asymmetric heating," Int. J. Heat Mass Transf., vol. 47, no. 25, pp. 5609-5612, Dec. 2004. 